\title{
Occult pneumothorax, revisited
}

\author{
Hesham R Omar ${ }^{1,2^{*}}$, Hany Abdelmalak ${ }^{1}$, Devanand Mangar ${ }^{2,3}$, Rania Rashad ${ }^{4}$, Engy Helal ${ }^{5}$, Enrico M Camporesi ${ }^{2,3,6}$
}

\begin{abstract}
Pneumothorax is a recognized cause of preventable death following chest wall trauma where a simple intervention can be life saving. In cases of trauma patients where cervical spine immobilization is mandatory, supine AP chest radiograph is the most practical initial study. It is however not as sensitive as CT chest for early detection of a pneumothorax. "Occult" pneumothorax is an accepted definition of an existing but usually a clinically and radiologically silent disturbance that in most patients can be tolerated while other more urgent trauma needs are attended to. However, in certain patients, especially those on mechanical ventilation (with subsequent increase of intrapleural air with positive pressure ventilation), missing the diagnosis of pneumothorax can be deleterious with fatal consequences. This review will discuss the occult pneumothorax in the context of 3 radiological examples, which will further emphasize the entity. Because a negative AP chest radiograph can dangerously delay its recognition, we recommend that any trauma victim presenting to the emergency department with symptoms of respiratory distress should be screened with either thoracic ultrasonography or chest $\mathrm{CT}$ scan to avoid missing a pneumothorax.
\end{abstract}

\section{Introduction}

The entity of occult pneumothorax is more frequently recognized nowadays due to the the increasing diffusion of CT scan and thoracic ultrasonography in the evaluation of trauma patients. These diagnostic tools enabled us to detect small abnormalities not clinched by the traditional chest radiograph. The concept of occult pneumothorax has been thoroughly discussed amongst the literature [1-5]. In trauma patients, AP chest radiograph has been traditionally the initial diagnostic imaging study especially if a cervical-collar limits patient mobilization. With advent of the extended FAST examination (Focused Assessment with Sonography for Trauma), most centers now utilize eFAST before the initial screening chest Xray. This review describes the differences between AP chest radiograph and chest $\mathrm{CT}$ in early detection of a pneumothorax in a trauma patient.

\section{Definition}

Occult pneumothorax is a pneumothorax that was not suspected clinically nor was evident on the plain radiograph but rather identified on computed tomography scan.

\footnotetext{
* Correspondence: hesham_omar2003@yahoo.com

'Departement of Internal Medicine, Mercy Hospital and Medical Center,

Chicago, Illinois, USA

Full list of author information is available at the end of the article
}

Due to the increased utilization of CT chest and thoracic ultrasonography as the initial screening tests for thoracic and abdominal trauma, occult pneumothorax has been common. Most nonradiologists diagnose pneumothorax based on the visualization of a superior-lateral visceral pleural stripe on the upright chest radiograph. This is however not feasible in the supine chest radiograph unless there is a sizable pneumothorax. Unfortunately, because of clinical concerns in trauma patients regarding cervical spine immobilization, initial imaging in seriously injured patients typically consists of a supine AP chest X-ray that is insensitive for detecting pneumothorax. As illustrated in figure 1 after an initial AP chest radiograph failed to show evidence of pneumothorax, a CT chest performed immediately after the chest Xray revealed right-sided pneumothorax.

\section{Incidence}

The reported incidence of occult pneumothorax varies widely between $3.7 \%$ in injured children presenting to an emergency department to $64 \%$ in intubated multi-trauma patients [6-8]. However, most publication agreed with an average incidence of $5 \%$ for all trauma patients. This highlights the inadequacy of the supine AP chest X-ray as compared to chest CT in detection of a post-traumatic pneumothorax. The incidence depends on the type of trauma, whether blunt or penetrating. In patients with 


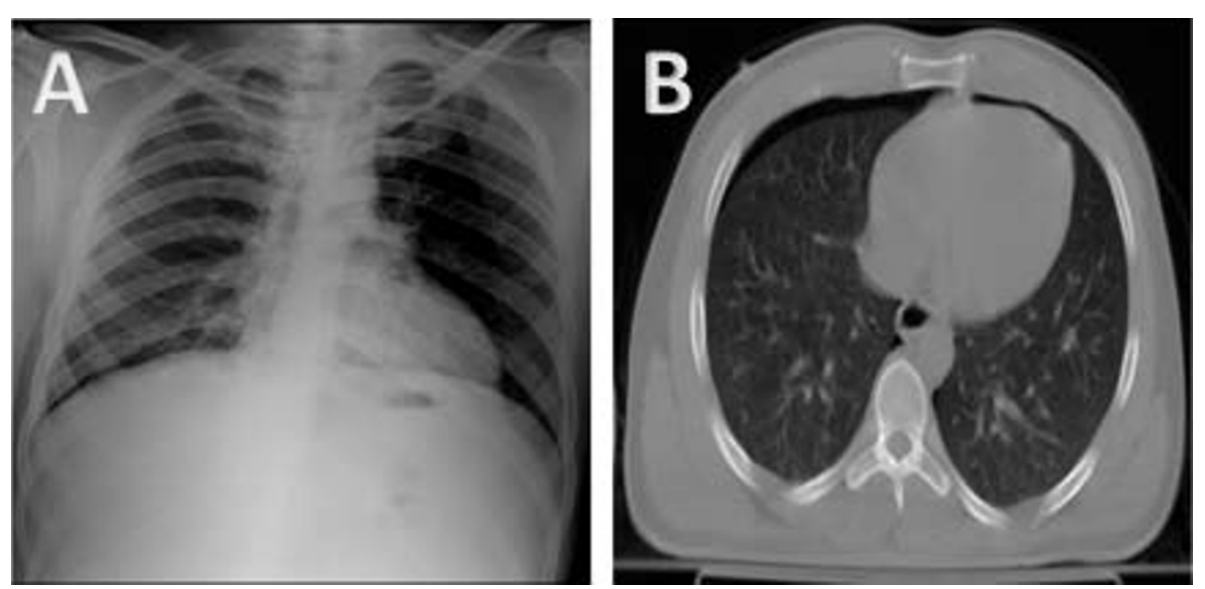

Figure 1 AP chest X-ray revealing no evidence of pneumothorax (Panel A). CT chest performed immediately after X-ray revealed right sided pneumothorax (Panel B).

blunt trauma the incidence of occult pneumothorax ranges between $2 \%$ and $15 \%$ depending on whether all patients in a given registry, or only those that underwent $\mathrm{CT}$, are included. This value may increase when the trauma team does the interpretation of the chest radiograph. In a retrospective study conducted by Wilson et al, [9] 1881 consecutive blunt trauma patients over a 102 month period were included. 307 patients developed pneumothoraces of which 68 were occult $(22 \%)$. The frequency of occult pneumothoraces in victims of penetrating trauma approaches $17 \%$. This was described in a recent level-III study of 5552 admissions at Grady Memorial Hospital [10].

Moreover, the reported proportion of pneumothoraces that are occult compared with those actually present on supine AP chest radiograph is variable and ranges from $29 \%$ to $72 \%[1-4,11]$. This variability is probably due to the fact that in some studies it is not always a certified and well-experienced radiologist who is responsible for making the diagnosis, which is the most precise way to identify the true incidence. A 17 month prospective study performed by Ball et. al. [12] concluded the incidence of occult pneumothorax to be as high as $76 \%$ when the radiographs are interpreted by the trauma team. This is higher than previously reported values in retrospective studies and is likely due to the difficult conditions in which the trauma team functions. Another factor that affects the variability in calculating the incidence of occult pneumothorax is whether anteroposterior chest radiograph or erect chest Xray is used. The reported sensitivity of erect chest X-ray vs. AP chest $\mathrm{X}$-ray in detecting occult pneumothorax is $92 \%$ and $50 \%$ respectively. This highlights the inadequacy of supine AP chest radiography for detecting pneumothorax and the requirement for a thoracic $\mathrm{CT}$ or ultrasonography to rule out the diagnosis in trauma patients.

\section{Why Occult?}

In the upright position, the classic sign for the diagnosis of pneumothorax is the visceral pleural line, which is visible as a thin curvilinear opacity along the lung and is separated from the chest wall by air in the apical pleural space. This sign is rarely identifiable on radiographs of supine patients unless there is a sizable pneumothorax. Small to moderate sized pneumothoraces may easily escape detection in that position.

In the supine patient, the least dependant pleural spaces are the anteromedial and subpulmonic recesses. Accumulation of air is expected in these 2 spaces initially with further extension laterally and apically as air volume increases or as the patient position becomes more upright. Therefore free air travels first to the highest region of the thorax; the cardiophrenic region. Larger volumes of free air then extend to the subpulmonic region inferiorly and to the anteromedial region superiorly [13]. These sites are easily missed on the conventional anteroposterior chest radiograph and indicate that the gold standard for ruling out pneumothorax is a thoracic CT scan.

Other than the body position, another determinant influencing the distribution of pleural air is an alteration in lung recoil due to consolidation or adhesion [14]. For example, in cases of postoperative left lower lobe collapse due to single lung ventilation (if the tube is advanced into the right main bronchus); the development of pneumothorax in these patients will usually have a posteromedial distribution [14]. Similarly, in patients with obliterated pleural areas due to adhesions, air will be excluded from these spaces.

Occult pneumothorax is concerning because of the risk of rapid progression to tension pneumothorax with positive pressure ventilation especially in trauma patients who have diminished cardiopulmonary reserve. 
Furthermore, clinical respiratory distress in these patients may be masked by sedation and concomitant respiratory support.

\section{Radiographic clues for suspicion of occult pneumothorax}

In supine or semi-erect patients, pneumothoraces do not appear in their classically described location over the apex and lateral to the lung. The insensitivity of the AP chest radiograph in the diagnosis of pneumothorax in supine patients has invited research looking for other possible clues for suspecting the diagnosis. The clues that help in raising the suspicion of pneumothorax in the AP chest radiograph if the classic signs are not evident include: [15-19]

1 . The high visibility of the cardiophrenic sulcus.

2. The deep sulcus sign which represent lucency of the lateral costophrenic angle extending toward the hypochondrium

3. Depression of the ipsilateral hemidiaphragm due to increased intrapleural pressure.

4. Double-diaphragm appearance due to air outlining of the anterior costophrenic angle and aerated lung outlining the diaphragmatic dome.

5. Improved sharpness of the cardiomediastinal border with a distinct cardiac apex due to anteromedial collection of air, which may appear as lucency. A sign known as crisp cardiac silhouette.

6. Increased sharpness of the pericardial fat pads, which become rounded and lobulated in the presence of air in the pleural space because they are no longer flattened by contact with the adjacent lung.

7. Visible inferior border of a collapsed lower lobe. A thin, sharp line may be detected which represents the inferior surface of the lung (visceral pleura) elevated and outlined by the inferior pneumothorax.

8. A band of air in the minor fissure bounded by two visceral pleural lines.

9. Visible lateral edge of the right middle lobe due to medial retraction in the presence of anterior pneumothorax.

Knowledge of these clues is mandatory for suspecting pneumothorax on AP chest X-ray. Radiologists, ER and critical care physicians should be aware of these signs to avoid missing a pneumothorax.

\section{Is occult pneumothorax predictable?}

Yes, there are several clinical markers that can predict for an increased incidence of pneumothorax even if not detected on the initial anteroposterior chest radiograph. These markers should be well known to emergency and ICU physicians for early suspecting the diagnosis. A level III retrospective study [20] with a purpose to determine the incidence, predictors, and outcomes for occult pneumothorax after trauma concluded that the presence of subcutaneous emphysema, pulmonary contusions, rib fractures and female sex were crudely associated with the presence of occult pneumothorax with an odds ratio of 5.47 for subcutaneous emphesema, 3.25 for pulmonary contusions and 2.65 for rib fractures. Although only $16 \%$ of patients with occult pneumothorax had subcutaneous emphysema, $98 \%$ of the patients with subcutaneous emphysema had an underlying pneumothorax whether overt $(82 \%)$ or occult $(16 \%)$. Therefore subcutaneous emphesema has a very high specificiy for diagnosis of occult pneumothorax but its absence is insufficient to rule out the diagnosis. Four years later, on a subsequent prospective level-II study [12] performed by the same group, only subcutaneous emphysema remained the only independent risk factor that can predict occult pneumothorax. Other risk factors including patient's age, mechanism of injury, intubation status in the emergency department, seat belt use, GCS score and the presence of pre-exisisting pulmonary comorbidities showed no significant predictive value for occult pneumothorax. We suggest that any trauma patient presenting with subcutaneous emphesema, pulmonary contusion or rib fractures should be further evaluated with CT chest to exclude any underlying pneumothorax not visible on the regular chest radiograph. Figure 2 well demonstrates the predictability of occult pneumothorax. The presence of lung contusions and subcutaneous emphesema has prompted further evaluation with $\mathrm{CT}$ chest, which revealed a pneumothorax.

\section{Truly Occult or Missed Pneumothorax?}

In a retrospective review of 44 severely injured patients identified with OPTX, 3 groups of radiologists reviewed the images for signs of pneumothorax [21]. The first group of radiologists which comprised 3 board-certified radiologists confirmed the presence of actual pneumothorax in 9 patients. The second and third group, which comprised a single board certified radiologist, each confirmed the presence of actual pneumothorax in 10 and 4 patients respectively. The deep sulcus sign was by far the most common "missed" sign $(75-90 \%$ of the missed pneumothorax depending on the group). Only 1 sharpened cardiac silhouette and an actual missed pleural line were also observed as shown in table 1.

Furthermore, these additional PTX signs observed on retrospective review were not identified consistently in a given patient across radiologist groups. When one considers this inconsistency among highly trained radiologists, with the reality that supine AP chest X-ray are typically first interpreted by non-radiologist trauma surgeons, the utility of this imaging modality is unclear. These secondary signs are often quite subtle and are rarely used by the clinicians making acute therapy decisions in the trauma bay. As previously mentioned, in 


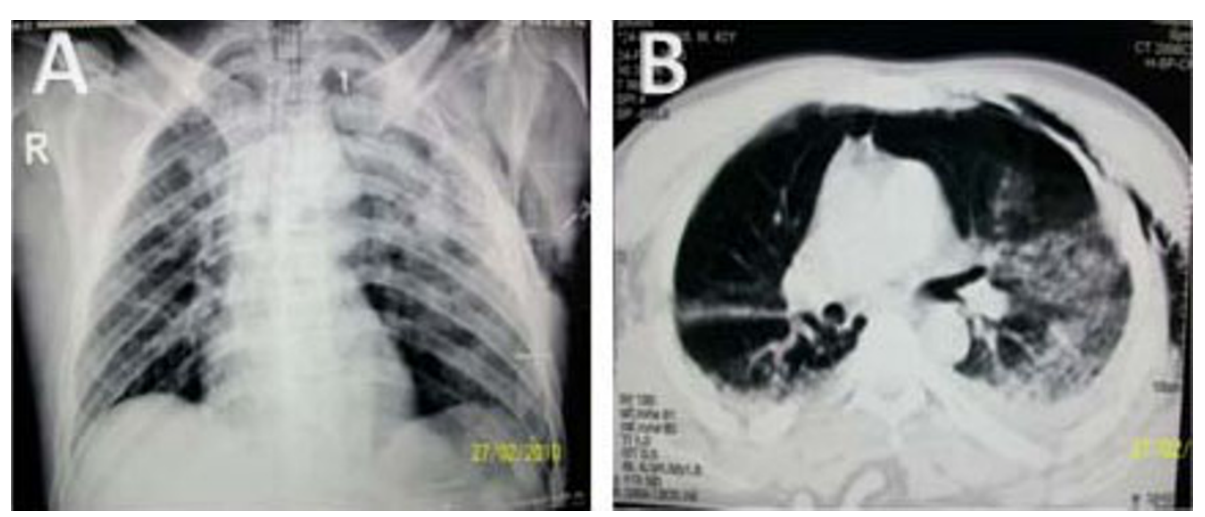

Figure 2 AP chest X-ray revealing evidence of bilateral lung contusions and left subcutaneous emphesema (Panel A). Chest CT confirmed both the lung contusions and the subcutaneous emphesema and demonstrated a left sided pneumothorax not initially appearing on the anteroposterior chest Xray (Panel B).

the 17-month prospective level-II study, the incidence of occult pneumothoraces appeared as high as $76 \%$ when interpreted by the trauma service at the time of admission [12]. Although the team may have good interpretive skills, they do not routinely have the luxury of prolonged interpretation times, a perfectly lit environment and premium digital monitors.

Figure 3 well illustrates how pneumothorax can present with subtle radiographic finding and therefore leading to a missed diagnosis. Notice the visible cardiophrenic sulcus and the apical pleural stripe in the AP chest radiograph. These were missed during interpretation of the Chest X-ray and could have lead to deleterious effects if passed un-noticed.

\section{Thoracic ultrasonography for early detection}

Although CT lung scanning is presently considered to be the standard diagnostic imaging for Pneumothorax, it has some disadvantages, including the need for patient transportation (which is not usually feasible in the unstable patient) and high doses of radiation. Lung ultrasonography has emerged in the past decade as a new and sensitive technique in the evaluation of respiratory diseases with a sensitivity of detecting pneumothoraces ranging from $92 \%$ to $100 \%$ among patients with blunt injuries [22-25]. Other advantages include the fact that it can be easily and quickly performed at bedside by a wide range of "sonographers," such as trauma, emergency, and critical care physicians [25]. The possible role played by chest ultrasonography in detecting the size and extension of a PTX is a challenging task which would allow the emergency department physician to take interventional decisions, such as the positioning of a chest tube, without wasting time. Potential pitfalls for thoracic ultrasonography such as the presence of pleural adhesions and emphysematous bullae
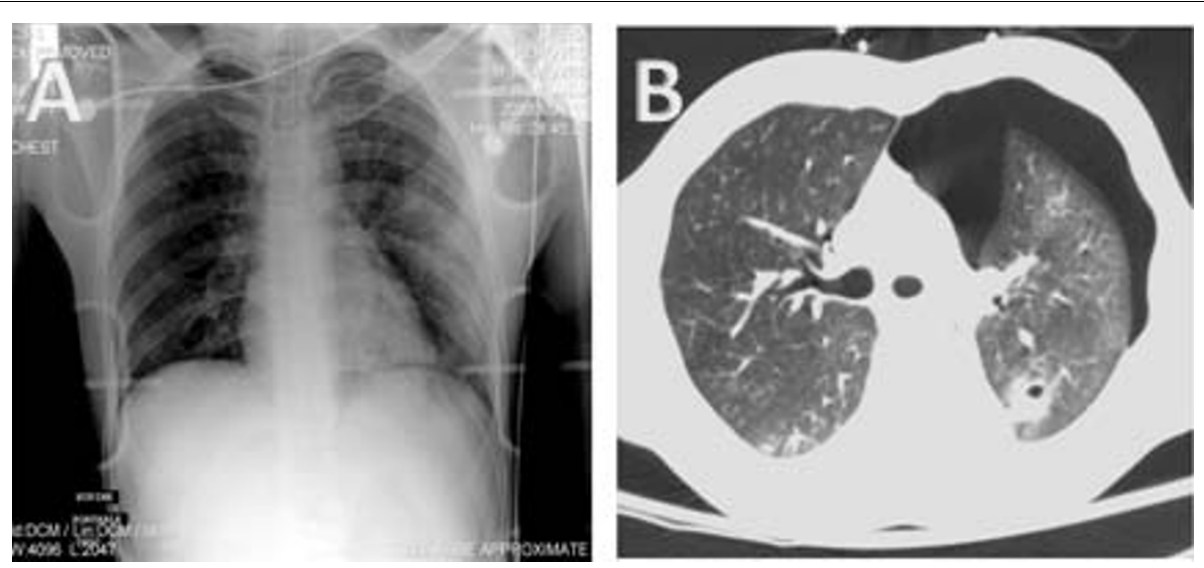

Figure 3 AP chest X-ray of the intubated patient, illustrating diffuse air space opacities in the left lower lung field (Panel A). Underlying pneumothorax was suggested because of a visible pleural stripe in the lung apex and a visible cardiophrenic sulcus. Chest $C T$ scan illustrating a left-sided pneumothorax with underlying lung collapse (Panel B). 
Table 1 Secondary Signs of PTXs Identified on Supine AP chest Xray

\begin{tabular}{lccc}
\hline & Group 1 & Group 2 & Group 3 \\
\hline Deep sulcus & 7 & 9 & 3 \\
\hline Crisp cardiac silhouette & 1 & 1 & 0 \\
\hline Pleural line & 1 & 0 & 1 \\
\hline
\end{tabular}

Adapted from Ball et al. from reference [21].

are less common in trauma patients. Therefore ultrasonographic evaluation of the thorax should be performed during the primary survey as a part of the eFAST examination for trauma patients [26]. This will identify a significant number of radio-occult Pneumothoraces and allow for sonar-guided interventions without exposing the unstable patients to the hazards of transportation and ionizing radiation.

\section{Management}

Clinicians appear to have greater difficulty deciding the appropriate therapy in patients with occult pneumothorax. The choice between close observation vs. early intercostal tube placement is still debatable, with reports of more complications from chest tube placement than from the pnemothorax, as long as the team remains aware of the pneumothorax. The traditional management of the majority of post-traumatic pneumothoraces detected clinically, or on chest X-ray, has been the placement of a chest tube. Tube thoracostomy is associated with up to $22 \%$ rate of major complications [27]. These include insertional (intercostal artery or intraparenchymal lung injuries), positional (requiring reinsertion) and infective (empyema or wound infection) issues [28-33]. A suggested algorithm for diagnosis and management of occult pneumothorax has been thoroughly described by Ball et. al. This algorithm utilized early thoracic ultrasound to limit CT over-usage [34].

In some patients, expert opinions support that close observation is safe, provided that the patient is not mechanically ventilated and that the pneumothorax is not increasing in size $[3,4,35,36]$. The importance of therapy is more relevant in the intubated patient on positive-pressure ventilation, as they are at increased risk of complications. However, the literature does not definitively identify which subset of patients receiving mechanical ventilation should receive or safely avoid tube thoracostomy.

Some authors believe that the risk of progression of a known pneumothorax to a tension pneumothorax is significant and that prophylactic chest tube placement for any patient with an occult pneumothorax subjected to positive-pressure ventilation is necessary $[10,37,38]$. And since the size of the initial occult pneumothorax is not predictive of progression or formation of a tension pneumothorax, therefore it cannot be used as a guide for placing a chest tube [30]. With all these conflicting results, the appropriate management for occult pneumothorax is still unsettled.

\section{Conclusion}

In conclusion thoracic CT scan is the "gold standard" for early detection of a pneumothorax, and is the imaging modality of choice for seriously injured blunt trauma patients. In centers utilizing eFAST technology for trauma victims, this should be the initial diagnostic modality. Its high sensitivity approaching $100 \%$, its availability at bedside without the need for transporting an unstable patient and its low profile for radiocarcinogenesis makes it an ideal tool. If not feasible, then we recommend that any trauma victim presenting to the emergency department with symptoms of respiratory distress should be offered a chest CT scan even if the chest X-ray showed no abnormalities.

This represents an in-depth illustrated review that will help understanding the entity of occult pneumothorax. The review is instructive for several reasons. First, it highlights the importance of the X-ray being interpreted by both a qualified radiologist and the ER or ICU physician to avoid missing an existing pneumothorax. Second, it demonstrates the predictability of pneumothorax in cases presenting with surgical emphesema, rib fractures or lung contusions. Third, it lists the different clues in the AP chest radiograph performed for the supine patient that can induce suspicion of an underlying pneumothorax if the classic signs are not present. Fourth, it emphasizes how the delay in diagnosing pneumothorax can dangerously affect the outcome and fifth, it emphasizes the importance of performing a thoracic ultrasound or a computed tomography of the chest following an initial negative AP chest radiograph in trauma victims with signs of respiratory distress especially those who will receive positive pressure ventilation.

\section{Consent}

Written informed consent was obtained from the patient's relatives for publication of this review article. A copy of the written consent is available for review by the Editor-in-Chief of this journal.

\footnotetext{
Author details

${ }^{1}$ Departement of Internal Medicine, Mercy Hospital and Medical Center, Chicago, Illinois, USA. ²Department of Anesthesiology/Critical Care; Tampa General Hospital; Tampa, FL, USA. ${ }^{3}$ Florida Gulf to Bay Anesthesiology, Tampa, FL, USA. ${ }^{4}$ Critical Care Department, Cairo University Hospital, Cairo, Egypt. ${ }^{5}$ Emergency Department, Elagouza Hospital, Cairo, Egypt.

${ }^{6}$ Department of Surgery/Anesthesiology, University of South Florida, Tampa, $\mathrm{FL}$, USA.
}

Authors' contributions

$\mathrm{HO}$ was responsible for literature search and drafting the manuscript and providing the explanatory figures. HA, EC and DM, RR and $E H$ have made 
critical revisions to the manuscript. All authors have read and approved the whole manuscript.

\section{Competing interests}

The authors declare that they have no competing interests.

Received: 14 April 2010 Accepted: 29 October 2010

Published: 29 October 2010

\section{References}

1. Neff MA, Monk JS, Peters K, Nikhilesh A: Detection of occult pneumothoraces on abdominal computed tomographic scans in trauma patients. J Trauma 2000, 49:281-285.

2. Ball CG, Hameed SM, Evans D, Kortbeek JB, Kirkpatrick AW, Canadian Trauma Trials Collaborative: Occult pneumothorax in the mechanically ventilated trauma patient. Can J Surg 2003, 46:373-379.

3. Brasel KJ, Stafford RE, Weigelt JA, Tenquist JE, Borgstrom DC: Treatment of occult pneumothoraces from blunt trauma. J Trauma 1999, 46:987-991.

4. Hill SL, Edmisten T, Holtzman G, Wright A: The occult pneumothorax: an increasing entity in trauma. Am Surg 1999, 65:254-258.

5. Silvani P, Colombo S, Cabrini L, Giudici D, Gallioli G, Torri G: Conventional radiology and computerized axial tomography in the diagnosis of pneumothorax in intensive therapy. Retrospective study of 2 years of activity. Minerva Anestesiol 1993, 59(9):427-33.

6. Guerrero-Lopez F, Vasquez-Mata G, Alcazar-Romero P, FernandezMondejar E, Aguayo-Hoyes E, Linde-Valverde CM: Evaluation of the utility of computed tomography in the initial assessment of the critical care patient with chest trauma. Crit Care Med 2000, 28:1370-1375.

7. Voggenreiter G, Aufmkolk M, Majetschak M, Assenmacher S, Waydhas C, Obertacke U, Nast-Kolb D: Efficacy of chest computed tomography in critically ill patients with multiple trauma. Crit Care Med 2000, 28:1033-1039.

8. Kirkpatrick AW, Sirois M, Laupland KB, Liu D, Rowan K, Ball CG, Hameed SM, Brown R, Simons R, Dulchavsky SA, Hamilton DR, Nicolaou S: Hand-held thoracic sonography for detecting post-traumatic pneumothoraces: The extended focused assessment with sonography for trauma (EFAST). J Trauma 2004, 57:288-295

9. Wilson H, Ellsmere J, Tallon J, Kirkpatrick A: Occult pneumothorax in the blunt trauma patient: tube thoracostomy or observation? Injury 2009, 40(9):928-31.

10. Ball CG, Dente CJ, Kirkpatrick AW, Shah AD, Rajani RR, Wyrzykowski AD, Vercruysse GA, Rozycki GS, Nicholas JM, Salomone JP, Feliciano DV: Occult pneumothoraces in patients with penetrating trauma: Does mechanism matter? Can J Surg 2010, 53(4):251-5.

11. Holmes JF, Brant WE, Bogren HG, London KL, Kuppermann N: Prevelance and importance of pneumothoraces visualized on abdominal computed tomographic scan in children with blunt trauma. J Trauma 2001, 50:516-520.

12. Ball CG, Ranson K, Dente CJ, Feliciano DV, Laupland KB, Dyer D, Inaba K, Trottier V, Datta I, Kirkpatrick AW: Clinical predictors of occult pneumothoraces in severely injured blunt polytrauma patients: A prospective observational study. Injury 2009, 40(1):44-7.

13. Tocino IM, Miller MH, Frederick PR, Bahr AL, Thomas F: CT detection of occult pneumothorax in head trauma. AJR Am J Roentgenol 1984, 143(5):987-90

14. Tocino IM, Miller MH, Fairfax WR: Distribution of pneumothorax in the supine and semirecumbent critically ill adult. AJR Am J Roentgenol 1985, 144(5):901-5.

15. Gordon R: The deep sulcus sign. Radiology 1980, 136:25-27.

16. Grainger RG, Allison DJ, Adam A, Dixon AK: Diagnostic radiology. New York, NY: Churchill Livingstone 2001, 254-257.

17. Armstrong $P$, Wilson AG, Dee $P$, Hansell DM: Imaging of diseases of the chest. St Louis, Mo: Mosby 2000, 770-771.

18. Tocino I, Armstrong J: Trauma to the lung. In Radiology. Edited by: Taveras J. Philadelphia, Pa: Lippincott-Raven; 1996:1-8.

19. Ziter FM Jr, Westcott JL: Supine subpulmonary pneumothorax. AJR Am J Roentgenol 1981, 137(4):699-701.

20. Ball CG, Kirkpatrick AW, Laupland KB, Fox DI, Nicolaou S, Anderson IB, Hameed SM, Kortbeek JB, Mulloy RR, Litvinchuk S, Boulanger BR: Incidence, risk factors and outcomes for occult pneumothoraces in victims of major trauma. J Trauma 2005, 59:917-25.
21. Ball CG, Kirkpatrick AW, Fox DL, Laupland KB, Louis LJ, Andrews GD, Dunlop MP, Kortbeek JB, Nicolaou S: Are occult pneumothoraces truly occult or simply missed? J Trauma 2006, 60:294-9.

22. Soldati G, Testa A, Sher S, Pignataro G, La Sala M, Silveri NG: Occult pneumothorax: diagnostic accuracy of lung ultrasonography in the emergency department. Chest 2008, 133(1):204-11.

23. Soldati G, Testa A, Pignartaro G, Portale G, Biasucci DG, Leone A, Silveri NG: The ultrasonographic deep sulcus sign in traumatic pneumothorax. Ultrasound Med Biol 2006, 32:1157-63.

24. Lichtenstein DA, Meziere G, Lascols N, Biderman P, Courret JP, Gepner A, Goldstein I, Tenoudji-Cohen M: Ultrasound diagnosis of occult pneumothorax. Crit Care Med 2005, 33(6):1231-8.

25. Kirkpatrick AW, Ball CG, Rodriguez-Galvez M, Chun R: Sonographic depiction of the needle decompression of a tension hemo/ pneumothorax. J Trauma 2009, 66(3):961.

26. Kirkpatrick AW, Sirois M, Laupland KB, Liu D, Rowan K, Ball CG, Hameed SM, Brown R, Simons R, Dulchavsky SA, Hamiilton DR, Nicolaou S: Hand-held thoracic sonography for detecting post-traumatic pneumothoraces: the extended focused assessment with sonography for trauma (EFAST). $J$ Trauma 2004, 57:288-95.

27. Ball CG, Lord J, Laupland KB, Gmora S, Mulloy RH, Ng AK, Schieman C, Kirkpatrick AW: Chest tube complications: How well are we training our residents? Can J Surg 2007, 50:450-8

28. Wall SD, Federle MP, Jeffrey RB, Brett CM: $C T$ diagnosis of unsuspected pneumothorax after blunt abdominal trauma. AJR Am J Roentgenol 1983, 141:919-21.

29. Tocino IM, Miller MH, Frederick PR, Bahr AL, Thomas F: CT detection of occult pneumothoraces in head trauma. AJR Am J Roentgenol 1984, 143:987-90.

30. Enderson BL, Abdalla R, Frame SB, Casey MT, Gould H, Maull Kl: Tube thoracostomy for occult pneumothorax: a prospective randomized study of its use. J Trauma 1993, 35:726-730.

31. Bridges KG, Welch $K$, Silver M, Schinco MA, Esposito B: CT detection of occult pneumothroaces in multiple trauma patients. J Emerg Med 1993, 11:179-86

32. Etoch SW, Bar-Natan MF, Miller FB, Richardson JD: Tube thoracostomy: factors related to complications. Arch Surg 1995, 130:521-6.

33. Bailey RC: Complications of tube thoracostomy in trauma. J Accid Emerg Med 2000, 17:111-4.

34. Ball CG, Kirkpatrick AW, Feliciano DV: The occult pneumothorax: what have we learned? Can J Surg 2009, 52(5):E173-9.

35. Collins JC, Levine G, Waxman K: Occult traumatic pneumothorax: immediate tube thoracostomy versus expectant management. Am Surg 1992, 58:743-746.

36. Wolfman NT, Myers MS, Glauser SJ, Meredith JW, Chen MY: Validity of CT classification on management of occult pneumothorax: A prospective study. AJR Am J Roentgenol 1998, 171:1317-1323.

37. Karnik AM, Khan FA: Pneumothorax and barotrauma. In Critical Care Medicine: Principles of Diagnosis and Management in the Adult. Edited by: Parillo JE, Dellinger RP. St. Louis: Mosby; , 2 2001:930-948.

38. Omert L, Yeaney WW, Protech J: Efficacy of thoracic computerized tomography in blunt chest trauma. Am Surg 2001, 67:660-667.

doi:10.1186/1752-2897-4-12

Cite this article as: Omar et al: Occult pneumothorax, revisited. Journa of Trauma Management \& Outcomes 2010 4:12. 